\title{
Criminologie
}

\section{Violence et répression : mythe ou réalité ?}

\section{Micheline Baril}

Volume 12, numéro 1, 1979

Justice et public

URI : https://id.erudit.org/iderudit/017100ar

DOI : https://doi.org/10.7202/017100ar

Aller au sommaire du numéro

Éditeur(s)

Les Presses de l'Université de Montréal

ISSN

0316-0041 (imprimé)

1492-1367 (numérique)

Découvrir la revue

Citer cet article

Baril, M. (1979). Violence et répression : mythe ou réalité ? Criminologie, 12(1), 66-77. https://doi.org/10.7202/017100ar d'utilisation que vous pouvez consulter en ligne.

https://apropos.erudit.org/fr/usagers/politique-dutilisation/ 


\section{VIOLENCE ET RÉPRESSION :}

MYTHE OU REALITÉ?

Micheline Baril

\section{Introduction}

Bien avant la naissance et la prolifération des sondages d'opinion publique, les gouvernements avisés avaient reconnu l'importance de l'opinion pour consolider et maintenir leur pouvoir, pour légiférer, \& surveiller et punir \$. Puisque la force, disait Hume, se situe toujours du côté des gouvernés, les gouvernants ne peuvent se maintenir en position de pouvoir que par l'opinion et ceci, même dans les régimes totalitaires. Pourtant, les lois et leur application ne sont jamais le reflet fidèle de l'opinion publique. Ce décalage peut s'expliquer de trois façons : l'opinion est difficile à cerner; il se peut qu'il n'y ait pas d'opinion sur un sujet donné ou qu'elle soit peu informée ; l'opinion peut être ignorée ou manipulée lors de la prise de décision. Pour un gouvernement soucieux de traduire dans ses politiques les volontés de ses ressortissants, les problèmes qui se posent sont donc ceux de la connaissance et de l'évaluation de l'opinion.

Quelles sont les volontés des ressortissants ? Le développement des statistiques et des techniques de sondage, depuis le siècle dernier, a suivi (et servi) le mouvement démocratique, renouvelant l'illusion d'un consensus et d'une participation populaire et créant une apparence de certitude dans le diagnostic. Du pifomètre des courtisans et conseillers de jadis aux grands sondages 《 scientifiques > d'aujourd'hui, le progrès semble énorme. Et pourtant, on peut se demander si on connaît réellement mieux l'opinion publique maintenant et si le sondage, à son stade actuel de développement, est en mesure de saisir cette opinion complexe et diversifiée, notamment en matière de crime et de justice.

Ce sont les résultats d'une recherche menée dans le cadre des travaux du Groupe de recherches du C.I.C.C. sur les attitudes envers la criminalité (G.R.A.C.) qui suggèrent de tels doutes. En effet, interrogés sur leurs opinions et attitudes à l'égard de la criminalité, les citoyens, en situation d'entrevue non directive, ont exprimé des vues très diversifiées, très nuancées, très ambivalentes et, pour le chercheur, difficilement réductibles en catégories bien tranchées de \& pour $>$ et de < contre 》. 
Les résultats ébranlent également une croyance vieille comme le droit pénal, un des fondements de ce droit, une de ses justifications, à savoir que le citoyen est un être essentiellement punitif dont l'instinct de vengeance doit être contrôlé par l'État qui, dès lors, punit au nom du citoyen. Si l'homme de la rétribution a facilement conquis criminologues et politiciens, l'homme de la compassion a été systématiquement méconnu. Parmi les raisons de cette méconnaissance, il faut souligner l'inégalité entre l'expert et le citoyen ordinaire en termes de responsabilité et d'information. Ce qui nous amène au deuxième point, celui de l'évaluation de l'opinion.

Dans quelle mesure l'opinion publique doit-elle, peut-elle guider les politiques sociales? Par exemple, le gouvernement canadien fait face, périodiquement, à des pressions populaires en vue de rétablir la peine de mort. Si tel est réellement (le problème de la connaissance de l'opinion se pose toujours) le vœu des citoyens, doit-on leur donner les têtes qu'ils réclament? En fin de compte, c'est le pouvoir qui assume la responsabilité des politiques qu'il adopte sur la base de jugements moraux et politiques. Il serait d'ailleurs dangereux de suivre aveuglément les courants de l'opinion publique car celle-ci est généralement très peu informée. C'est ce qui ressort de l'étude sur l'image de la violence : les citoyens connaissent peu et mal les phénomènes de la criminalité et de son contrôle. Les modalités d'intervention qu'ils favorisent reposent sur des visions très partielles, des mythes, des croyances, un folklore de la criminalité entretenu par les media et non démenti par ceux qui possèdent une connaissance plus vaste. Paradoxalement, elles s'appuient aussi sur l'existence des lois et des organismes de contrôle. Et si les citoyens s'accommodent bien de telle loi, de telle forme d'intervention, rien ne dit que, devant le fait accompli ou en présence d'informations pertinentes, ils ne s'accommoderaient pas tout aussi bien d'une autre loi ou d'une absence d'intervention. À moins qu'on ne parvienne à identifier les valeurs fondamentales, les attitudes de base des citoyens, leurs opinions seront de faible utilité pour l'élaboration de politiques criminelles efficaces.

Mais j'anticipe. Ces considérations sur la mesure de l'opinion et sur le mythe des citoyens répressifs m'ont été inspirées par le projet de recherche que je décrirai brièvement et dont je résumerai les résultats. 


\section{Le projet de recherche}

Il s'agit d'une recherche exploratoire qualitative, conduite durant l'été 1976, auprès de 54 Québécois, par la méthode d'entrevues non structurées, focalisées sur le thème de la violence.

L'échantillon a été constitué selon une technique non probaliste, dans la perspective d'une diversité maximale sur des variables, tels le lieu de résidence, le sexe, l'âge, le statut socio-économique, l'occupation, l'origine ethnique et la connaissance personnelle de la criminalité de violence (i.e. citoyens victimes et non victimes, policiers, intervenants professionnels).

C'est une technique d'entrevue non directive qui a été choisie, l'interviewer visant non pas à une impossible neutralité mais à un climat naturel qui favorise l'expression. L'objectif de la recherche étant d'explorer le domaine des perceptions, opinions et attitudes des Québécois face à la violence et à son contrôle, les entrevues étaient focalisées sur ces thèmes. On aura remarqué que le point central de l'étude était la violence, qu'elle soit ou non définie comme criminelle. En effet, dans une étude préliminaire, il semblait opportun de se situer non pas au niveau des définitions légales mais sur le plan des réalités vécues et des jugements individuels. Cette définition permettait, du moins l'espérions-nous, une meilleure compréhension des seuils de tolérance de même que des attentes vis-à-vis de la criminalité ou de la décriminalisation de certains actes. En outre, cette façon de procéder constituait un moyen additionnel pour élaborer une définition de la violence, définition suggérée par le public québécois et, éventuellement, canadien.

\section{Les résultats}

\section{a) La violence}

On va dire la violence, c'est la violence sociale, puis la violence de ci, puis de ça, mais qu'est-ce que c'est la violence? Violence, si je m'arrête à y penser, en autant que je suis concerné, moi je ne peux pas définir le mot violence, d'une façon claire et nette la, comme une chaise, c'est une chaise, un chat, c'est un chat, c'est tout.

La violence a été définie comme un concept relatif, émotivement chargé, à connotation péjorative et particulièrement diffi- 
cile à cerner. Les personnes interviewées l'ont abordée sous trois angles : du point de vue des auteurs :

C'est faire du mal à quelqu'un.

du point de vue des victimes :

La violence, c'est ce que les autres nous font. [...] C'est endurer.

et comme mode d'interaction :

Pour moi, c'est une réponse, ... une réponse disproportionnée, de l'excès.

On a identifié plusieurs sortes de violences qu'on pourrait résumer à deux types : la violence criminelle traditionnelle et la violence sociale. A l'intérieur du premier type, il existe un consensus pour définir quelques actes comme violents par nature (meurtre, viol...). Par contre, les opinions varient quant au caractère violent ou non de plusieurs infractions (le cambriolage ou le vandalisme, par exemple) et des conduites relevant du domaine de la violence sociale. Sous ce dernier type, les interlocuteurs ont relevé des phénomènes tels que les guerres, les contestations, certaines actions syndicales, l'exploitation économique par les puissants, etc.

Les crimes sans victimes ne sont pas inclus au nombre des comportements violents de même qu'un certain nombre de crimes contre la personne : prise d'otages ou enlèvements, avortement ou, encore, l'incendie volontaire.

L'intention de l'agresseur et le consentement de la victime constituent les deux critères les plus importants pour juger de la violence d'une conduite :

Le médecin, c'est pour guérir, c'est différent.

... tordre des bras dans une cellule de police où c'est un policier qui fait ça à... un présumé bandit, bien à ce momentlà, je pense que c'est de la violence parce que, bon, le bandit, ordinairement, ne participe pas à... ou ne prend pas ça comme un plaisir.

Le contexte d'un comportement peut en modifier entièrement la nature ; en général, les situations auxquelles on peut s'identifier, celles qui sont familières, sont rarement définies comme très violentes, une certaine distance étant nécessaire pour accoler les qualificatifs « violent \$ ou * dangereux , à des actes. La violence 
du contrôle social, formel ou informel, est rarement reconnue comme telle et, lorsqu'elle l'est, elle semble légitime et désirable.

Enfin, il faut noter que, chez les interlocuteurs, la violence ne fait pas toujours l'objet d'une réprobation et qu'il n'existe pas une concordance parfaite entre le degré de gravité attribué à une conduite et l'intensité de la condamnation dont elle fait l'objet.

b) La peur du crime

Si on définit la peur du crime comme la crainte d'être personnellement victimisé, il faut mettre en doute les résultats des sondages effectués jusqu'ici, lesquels concluent à une peur généralisée dans la population.

Non... pas peur. Il m'est jamais rien arrivé. Ah ! bien c'est comme d'autres événements, c'est des choses qui arrivent... Comme des meurtres, il y en a, on en a relevé deux cents par année. Sur six millions... pour être là, ça prendrait bien un drôle de hasard...

Seules les victimes récentes d'actes perçus comme très violents exprimaient des craintes concrètes et avaient modifié leurs habitudes en conséquence.

Depuis ce temps-là, je ne suis pas sortie une fois toute seule, le soir. J'ai peur que cela se reproduise et que, à ce momentlà, je ne sois pas capable de me défendre.

Pour les autres interlocuteurs, la peur du crime revêt des significations autres que la crainte d'être victime. Les situations les plus rares sont celles qu'on redoute davantage : l'attaque imprévisible, dans la rue, par un étranger ; et de manière générale, celles où l'on ressent une certaine vulnérabilité. De plus, l'augmentation actuelle et future de la violence est source d'une grande anxiété.

On a trop souvent interprété l'expression de sentiments forts à l'égard de la violence comme une crainte personnelle de victimisation. Sous ce rapport, nos données sont claires et révélatrices. Les interlocuteurs abhorrent la violence physique grave, mais à moins d'en avoir déjà été victimes, ils se sentent plus ou moins immunisés contre elle.

c) Les jugements et les sanctions

Le plus souvent, les comportements les plus violents sont aussi jugés comme les plus graves .Les actes qui portent atteinte 
à l'intégrité physique ou morale des personnes sont plus graves que ceux qui sont dirigés contre la propriété. Sous la rubrique " violence criminelle traditionnelle , les comportements jugés violents par l'ensemble de l'échantillon suscitent également un consensus au niveau de la gravité.

Toutefois, les circonstances dans lesquelles se produit un acte en atténuent ou en augmentent la gravité. Dans leur évaluation de conduites spécifiques, les interlocuteurs prennent en considération des éléments tels que le mode de perpétration, l'intention et les caractéristiques des agresseurs :

Une femme qui va tuer son mari peut toujours plaider que c'était à son corps défendant, ça se peut. Un homme qui tue sa femme, c'est malaisé qu'il plaide que ce fut à son corps défendant.

Les conséquences des actes, leur caractère délibéré ou spontané :

Ce n'est pas pareil et quand un gars fait un meurtre sur un coup de tête ou sì c'est la rage ou quand ça fait longtemps qu'il y pense.

Les caractéristiques de la victime : son statut, son caractère moral, ses attitudes et la dynamique de sa relation avec l'agresseur constituent des facteurs atténuants ou aggravants.

Une attitude de tolérance prévaut vis-à-vis de l'agression entre proches parents ou amis. Même lorsqu'il s'agit de meurtre, comportement le plus grave, l'agresseur est compris, voire même excusé, et est considéré comme ayant droit à la clémence. Les propos des interlocuteurs suggèrent que les liens de parenté ou d'amitié ne diminuent pas nécessairement la gravité de l'acte mais ils entraînent une certaine clémence au niveau de la réaction.

En somme, il est rare que la seule gravité de l'acte, en soi, détermine un désir ou une action stigmatisante. Il semble que les deux principaux déterminants de la tolérance au niveau de l'évaluation des actes soient l'identification à l'agresseur (plutôt qu'à la victime) et la qualité de l'information sur la criminalité et le système de justice criminelle.

En somme, si l'on tient compte de tous les « si >, 《 mais », (à moins que \$, a à condition que », de tous les cas spéciaux et de toutes les restrictions apportées par les Québécois interviewés, bien peu d'actes de violence sérieuse seraient entièrement condam- 
nables. Même l'homicide, considéré comme la violence et le crime par excellence, est excusé, dans plusieurs circonstances.

De plus, les actes jugés les plus graves n'appellent pas nécessairement les sanctions les plus sévères. Des considérations diverses peuvent inspirer la clémence : improbabilité de la récidive, méfiance envers le système de justice criminelle, croyances relatives à la cruauté et/ou à l'inefficacité des châtiments. Par contre, il arrive qu'on préconise des peines relativement sévères pour des délits jugés mineurs, cette décision ayant pour objectif de marquer l'existence de la règle. De plus, certaines personnes sont fondamentalement opposées au châtiment, quelle que soit la gravité de l'acte ; elles ne s'y résignent qu'en dernier ressort et lorsque le danger apparaît imminent.

Relativement à la peine capitale on constate une grande ambivalence. On croit fermement en son pouvoir d'intimidation ; ceux qui recommandent l'abolition le font en dépit de cette croyance et pour des principes et motifs humanitaires; ceux qui en recommandent le maintien le font à cause de cette croyance et aussi, parfois, pour des motifs humanitaires (la détention à long terme leur apparaissant trop cruelle).

Ce qui est remarquable dans la façon dont les citoyens évaluent les conduites dangereuses ce sont les nuances du raisonnement à chaque palier de l'évaluation. Bien sûr, il y a des gens catégoriques, intransigeants, qui attribuent aux actes un caractère absolu de bien ou de mal. Le plus souvent, face à des actes concrets les interlocuteurs jugent de façon très pondérée et font preuve d'une grande tolérance. Le public juge selon les données dont il dispose. S'il en arrive à des verdicts erronés ou naïfs, ce n'est sûrement pas faute de critères de décision.

\section{d) Les mythes}

Il existe des croyances basées sur quelques éléments de réalité, démesurément amplifiés et ancrés dans l'esprit de la population, touchant la récidive (les crimes les plus graves sont commis par des récidivistes), le pouvoir intimidant de la peine et le criminel comme un être différent, fondamentalement, des honnêtes gens. Le mot « criminel » évoque l'image d'un être dénaturé, monstrueux, redoutable, une personne à qui on ne peut s'identifier. Peut-être est-ce la raison pour laquelle les victimes d'actes 
criminels violents avaient tendance à être plus tolérantes que les non-criminels : leurs agresseurs avaient forme humaine.

Alors que les crimes sensationnels d'actualité sont assez bien connus des hommes de plus de $\mathbf{3 0}$ ans, alors que quelques meurtres spectaculaires des années passées font partie du folklore de la criminalité, la majeure partie de la réalité criminelle échappe à la connaissance ou à la perception des citoyens. Ces derniers n'en démontrent pas moins d'intérêt et de souci de compréhension de même que, dans la grande majorité des cas, une propension marquée à la pondération.

Le fossé qui existe entre les opinions des spécialistes et celles des autres citoyens semble se situer au niveau de l'information et du vocabulaire. Le mot «criminel », par exemple, recouvre des réalités différentes.

\section{e) Le système de justice criminelle}

Sa fonction principale serait de protéger la société : assurer la sécurité et la qualité de la vie, maintenir l'ordre social et réaffirmer l'existence des normes. Ces buts seraient atteints surtout par le moyen de l'intimidation qui constitue la finalité première de la sanction pénale; la récidive étant vue comme particulièrement menaçante.

Dans l'ensemble, le système de justice apparaît comme plutôt sympathique, plutôt intègre mais largement inefficace. Dans ses fonctions de prévention et de répression de la criminalité, la police est évaluée positivement bien que son efficacité laisse à désirer. On appuie l'usage de la force par la police sauf dans de rares cas où elle paraît abusive :

Je ne suis pas contre qu'il y ait un peu de violence dans la police. Autrement, c'est les criminels qui ont toute la protection et les gens honnêtes paient tout le temps.

L'action policière, dans le champ de la criminalité violente traditionnelle est reconnue légitime et souhaitable lorsqu'on accepte le système social. Les citoyens ont alors l'impression de posséder leur police.

Par contre, l'intervention policière dans les conflits ou manifestations collectives fait l'objet d'un dissensus quant à son opportunité et, surtout, quant à la manière dont elle s'effectue. 
Enfin, l'inefficacité de la police serait due à son impuissance : impuissance face à des criminels puissants et bien protégés; impuissance face aux autres agences du système de justice criminelle qui agissent à l'encontre de l'action policière et des intérêts des citoyens.

Les tribunaux et les agences correctionnelles, pour leur part, projettent une image assez terne et stéréotypée. Ils sont peu connus et on les juge à leurs fruits, c'est-à-dire, selon les interlocuteurs, la récidive, l'augmentation de la violence et l'immunité accordée aux grands du monde interlope, pour conclure que, finalement, il n'y a pas de justice.

Chez les interlocuteurs qui avaient été victimes d'actes criminels violents, l'image était nettement plus négative. Ceux qui ont été appelés à se présenter devant un tribunal ont vécu cette expérience un peu comme une seconde victimisation ou comme une conséquence négative additionnelle. L'épreuve fut troublante, déconcertante et, parfois même, occasion de traumatismes.

On aurait dit que c'était moi, la criminelle. Je ne m'attendais vraiment pas à ça. [...] Je pensais que j'allais mourir là, bien franchement, je n'ai rien vu, tout était embrouillé, je voulais juste disparaître. C'était sale, sale, [...] Si c'était à refaire, je t'assure que je ne dirais pas un mot, pas un mot à personne. Je l'ai eue ma leçon.

Commentaire d'autant plus troublant qu'il n'est pas unique. Les victimes regrettent d'avoir porté plainte, en particulier lorsqu'un suspect est arrêté et une procédure judiciaire entamée.

Perte d'argent, humiliation, inutilité de la démarche. Et quoi encore? Perte de confiance dans le système.

A la cour, faut pas essayer de comprendre ; c'est du chinois. Ils se lancent des fleurs, puis tout à coup, ça tourne en bêtises. Ils te demandent toutes sortes de questions niaiseuses puis oup ! Ia cour est quelque chose...

Ton affaire est finie... C'est tout.

Quant aux autres agences, les seules qui aient été mentionnées sont les prisons et le service des libérations conditionnelles.

Les prisons apparaissent plutôt comme un mal nécessaire. Elles sont des écoles du crime mais, au moins, elles restreignent l'agir criminel, si ce n'est que temporairement. Le désir d'un 
traitement humanitaire des détenus apparaît couplé au désir non moins intense de vivre en toute quiétude.

Pour sa part, le service des libérations conditionnelles n'a trouvé aucun défenseur parmi nos interlocuteurs. Le service lui-même est complètement inconnu ; c'est le fait de libérer des individus avant terme qui fait l'objet de la désapprobation populaire. Non par principe, non par désir de rétribution, mais parce que les libérés conditionnels sont perçus comme des gens qui récidivent.

Enfin, il semble que ce que nous nommons « système de justice criminelle » ne soit pas perçu comme un tout, un système unifié par la poursuite d'un but commun mais comme un ensemble d'organismes dont les objectifs et les méthodes sont mutuellement incompatibles.

Je trouve que la justice est... les policiers eux-mêmes feraient assez bien leur travail. C'est après qu'ils les ont trouvés que les autres les lâchent. A ce compte-là, il n'y aurait pas besoin de justice du tout. S'ils arrêtent quelqu'un, puis qu'ils le lâchent tout de suite après, ils seraient bien mieux de ne pas l'arrêter du tout.

\section{Quelques commentaires sur la mesure de l'opinion}

Il a été question plus tôt des difficultés que pose la mesure de l'opinion. Une comparaison des résultats de l'étude sur l'image de la violence avec les conclusions de divers sondages portant sur les réactions au crime ${ }^{ \pm}$met en relief les faiblesses de l'approche quantitative telle qu'elle a été utilisée jusqu'ici.

a) La connaissance et la visibilité des objets investigués

La population est très mal informée et c'est peut-être là la limite la plus sérieuse au sondage. En ce qui concerne la criminalité, il faut rappeler que seuls les crimes les plus graves, les plus sensationnels, les plus exploités par la presse, étaient présents à l'esprit des interlocuteurs, et ceci, quels que soient leur âge et leur éducation. Leurs opinions en matière de justice, prévention et châtiment, sont donc teintées par cette vision partielle de la réalité. Vision fortement influencée, parce que si parcellaire, par les événements marquants de l'actualité : une émeute dans les

1. Ces sondages ont été présentés dans la 《Revue de littérature * qui fait l'objet du rapport no 2 du G.R.A.C. 
prisons, une vague de viols, un meurtre particulièrement sadique, une campagne pour la peine de mort.

Quant au système de justice criminelle, à l'exception de la police, il est encore moins visible que le crime. Les citoyens connaissent peu le fonctionnement des tribunaux. Si l'existence de prisons leur est connue, ils ignorent tout ou presque des régimes carcéraux ou de leurs effets (sauf comme écoles du crime). Lorsqu'un citoyen réclame de longues peines d'emprisonnement mais que, par ailleurs, il est convaincu que la vie est douce en prison, son opinion a une signification tout autre que celle de ce citoyen également en faveur des longues détentions mais qui considère la prison comme un lieu de châtiment très sévère.

Interrogés sur les nouvelles (été 1976) législations concernant la peine de mort et le contrôle des armes à feu, les Québécois n'avaient que de vagues notions des changements apportés ou proposés aux lois existantes que, d'ailleurs, ils connaissaient à peine. De la probation, ils ne connaissent que le nom. Le Service des libérations conditionnelles, tant redouté, ne signifie que la remise en liberté de criminels dangereux susceptibles de venir nous égorger puisqu'on leur donne carte blanche. Il s'agit, en effet, dans leur vision, d'une mesure anti-éducative. Comme la mère qui adoucit la punition imposée par le père, le Service des libérations conditionnelles invalide en quelque sorte la décision du juge.

b) Le vocabulaire du chercheur et celui des répondants

Au problème de la pauvreté d'informations se greffe celui du vocabulaire. Il semble, par exemple, que le terme «criminels » soit interprété par les citoyens non spécialistes des affaires criminologiques comme «vrais criminels», c'est-à-dire comme des êtres fondamentalement dépravés, dangereux, incorrigibles, tarés. Nous avons également vu les problèmes de définition que pose le concept «peur du crime ». Alors que les chercheurs ont tendance à interpréter une peur exprimée en termes de «crainte de victimisation », souvent les personnes interrogées ne manifestent que leur inquiétude morale ou leur désapprobation de conduites perçues comme potentiellement dangereuses.

\section{c) Les paliers d'évaluation}

Selon nos interlocuteurs, un acte violent n'est pas nécessairement répréhensible ou criminel ; un acte criminel est plus ou 
moins grave selon les circonstances qui l'entourent ; même grave, une conduite peut être excusée dans certaines situations; enfin, un crime grave et réprouvé peut faire l'objet de sanctions variées selon les images qu'on a des diverses interventions possibles.

Dans la mesure de l'opinion, on a généralement négligé de tenir compte des significations et des divers paliers d'évaluation des conduites. Or, les gens jugent de façon très différente selon qu'on leur présente une conduite hors contexte ou une situation concrète spécifique et selon qu'on les laisse s'exprimer à leur guise ou non.

\section{Une opinion publique répressive}

Criminologues comme politiciens s'appuient souvent sur la soif de vengeance, le besoin de rétribution et l'intolérance de l'opinion publique, pour expliquer des décisions qui, semble-t-il, ne correspondent pas entièrement à leurs propres visions. La voix populaire réclame que les déviances soient réprimées et punies. Bien malgré nous, diront ces criminologues et politiciens, l'écoutons-nous. Et, ce faisant, ils renforcent d'autant cette punitivité populaire dont on ne sait plus trop l'origine. Bien sûr, d'autres personnes, d'autres groupes y trouvent leur intérêt. Entretenir la peur, exciter l'imagination ont toujours été des activités lucratives. Il suffit de machiner une vague de crimes, de raconter des atrocités, de décréter des châtiments plus sévères, de promulguer une loi, pour déclencher une réaction sociale punitive. Mais est-ce bien une réaction punitive? La loi entraîne sa propre nécessité. La peur engendre la violence.

Les propos des Québécois rencontrés lors de l'étude sur l'image de la violence jettent des doutes sur cette « opinion publique répressive ». Les citoyens veulent vivre en sécurité et non châtier des transgresseurs. Ils demandent une société bien ordonnée, non une société répressive. Ils croient en la constance et en la certitude de la punition, mais croient-ils en la punition ? 